\title{
Self-Rotation Number using the Turning Angle
}

\author{
H. R. Dullin, D. Sterling, J. D. Meiss * \\ Department of Applied Mathematics, \\ University of Colorado, Boulder, CO 80309-0526
}

May 4, 2000

\begin{abstract}
The self-rotation number, as defined by Peckham, is the rotation rate of the image of a point about itself. Here we use the notion of "turning angle" to give a simplified algorithm to compute the selfrotation number for maps that "avoid an angle." We show that the orientation preserving Hénon map does avoid an angle. Moreover, the self-rotation number for orbits of the Hénon map can be computed once and for all at the anti-integrable limit by a simple algorithm depending upon the symbol sequence for the orbit.
\end{abstract}

AMS classification scheme numbers: 58F05, 58F03, 58C15

\section{Introduction}

The rotation number of an orbit of a map measures the average fraction of a circle through which the orbit turns per iteration of the map. It is most

${ }^{*}$ Useful conversations with R. Easton and B. Peckham are gratefully acknowledged. HRD was supported by DFG grant number Du 302, DS was supported in part by NSF traineeship grant number DMS-9208685, and JDM was supported in part by NSF grant number DMS-9971760 
easily defined for maps of the circle or of the annulus, where the topology of the space provides a natural angle.

More formally, one defines the rotation number of a circle map by lifting the angle variable from the circle to its covering space, the line. The map extends as well to a "lift", which is defined as a map on the covering space that gives the original dynamics when projected back to the circle [1]. For example, if $f$ is a map of the circle $S$, and $\theta$ represents the angle coordinate, we let $\tilde{\theta} \in \mathbb{R}$ be the lifted angle. Then if $\tilde{\theta} \bmod 2 \pi=\theta$, they represent the same physical point. A lift $\tilde{f}$ is any map such that

$$
\tilde{f}(\tilde{\theta}) \bmod 2 \pi=f(\tilde{\theta} \bmod 2 \pi)
$$

It is easy to see that any two lifts differ at most by some integer multiple of $2 \pi$. Given a lift, the rotation number of an orbit is defined as

$$
\rho(\theta)=\lim _{t \rightarrow \infty} \frac{\tilde{f}^{t}(\theta)-\theta}{2 \pi t}
$$

providing the limit exists. The rotation number of an orbit does depend upon the lift, but since lifts only differ by multiples of $2 \pi, \rho \bmod 1$ is independent of the lift.

An orbit of period $q$ always has a rational rotation number since there is always some integer $p$ such that

$$
\tilde{f}^{q}\left(\theta_{0}\right)=\theta_{0}+2 \pi p
$$

so that $\rho=p / q$. Note that $p$ is the integer number of times that the orbit winds around the circle, so that $\rho$ is the average number of times the orbit winds around the circle per iteration of the map. The rotation number of a periodic orbit does not change if the orbit persists under changes in parameter, because the integer $p$ cannot change.

For a map of the annulus, a lift is similarly defined in terms of the $\theta$ component of the map. For example if $\left(\theta^{\prime}, r^{\prime}\right)=f(\theta, r)$, then the first component, $f_{1}$, must be lifted to obtain a map on the strip. The fact that the rotation number on the annulus is an invariant is one key idea in the existence proof for ordered orbits in "twist maps" $[2,3]$. 
If the phase space is the plane, then one normally defines the rotation number relative to another orbit, such as a fixed point $x^{*}$. Removing $x^{*}$ from the plane leaves an annulus: $\mathbb{R}^{2} \backslash\left\{x^{*}\right\} \cong S \times \mathbb{R}^{+}$. In this case the rotation number measures the average rate of rotation around $x^{*}$. One problem with this definition is that in order to compute the rotation number of an orbit, one must do it "relative" to the fixed point. Moreover, this rotation number can change discontinuously upon continuous parameter changes even when there are no bifurcations. It is enough, for example, for the fixed point to move from inside to outside the convex hull of the orbit.

An alternative notion of rotation number that does not suffer this defect is the "self-rotation" number of Peckham [4], see $\S 3$. The self-rotation number of a periodic orbit is invariant under parameter changes in the map, so long as the orbit persists. However in order to compute this rotation number, one must explicitly construct a lift for certain polar coordinates defined for pairs of points in the phase space. This can be done by a continuation method, but is not computationally efficient.

In $\S 4$ we discuss an alternative construction of the self-rotation number based on the "turning angle," defined as the angle through which the vector from $x$ to its image $f(x)$ turns upon iteration. When this turning angle does not take all possible values on the circle, we say that the map avoids an angle. In this case we can obtain the self-rotation number without constructing a lift.

We will see in $\S 5$ that the quadratic diffeomorphism of the plane, the Hénon map, avoids an angle when it is orientation preserving. We will use the self-rotation number to classify periodic orbits of the Hénon map, and to find restrictions on the type of bifurcations that can occur. Moreover, we will see in $\S 5.5$ that the self-rotation number can be computed at the "anti-integrable" limit for orbits of the Hénon map, and we can associate a rotation number with the symbol sequence for orbits of the map.

We begin by using the turning angle to define rotation numbers for polygons. 


\section{Self-Rotation Numbers for Polygons}

Even if there is no underlying dynamical system, we can still compute rotation numbers for ordered sequences of points in the plane, that is, polygons. In this section we define two rotation numbers, the rotation number "relative to a point", and the "self-rotation number."

A plane polygon, $\mathcal{P}_{n}$, is a closed curve defined by an ordered sequence of $n$ vertices $x_{i} \in \mathbb{R}^{2}, i=0, \ldots, n-1$ that are connected by line segments $\left[x_{i}, x_{i+1}\right]$, see Fig. 1 . We assume that neighboring points of the polygon are distinct, but allow the sides to intersect. For convenience, we let $x_{n}=x_{0}$, and $x_{-1}=x_{n-1}$. Alternatively a polygon can be represented by the set of vectors that give its sides

$$
v_{i}=x_{i}-x_{i-1}, \quad i=0 \ldots n-1 .
$$

We require that $v_{i} \neq 0$, since otherwise the polygon does not have $n$ vertices. Note that only $n-1$ of these vectors are independent; the remaining variable, corresponding to $x_{0}$, say, represents the position of the polygon in the plane.

Let $\Phi_{\alpha}\left(v_{1}, v_{2}\right)$ be the angle between two nonzero vectors $v_{1}$ and $v_{2}$ in the plane, i.e.,

$$
\Phi_{\alpha}\left(v_{1}, v_{2}\right)=\arctan _{\alpha}\left(v_{1} \times v_{2}, v_{1} \cdot v_{2}\right),
$$

where $\tan \left(\arctan _{\alpha}(y, x)\right)=\frac{y}{x}$ and the $\arctan$ function is chosen to have the range $(\alpha, 2 \pi+\alpha]$. Perhaps the most natural choice would be $\alpha=-\pi$; however, we allow for other possible choices for our applications below.

We first consider the rotation number with respect to a "center" point $p$. This can be defined using the angle $\Phi_{\alpha}\left(x_{i}-p, x_{i+1}-p\right)$, the angle that the side $v_{i+1}$ subtends from $p$. The rotation number with respect to $p$ is the average subtended angle:

$$
\rho_{p}\left(\mathcal{P}_{n}, \alpha\right)=\frac{1}{2 \pi n} \sum_{i=0}^{n-1} \Phi_{\alpha}\left(x_{i}-p, x_{i+1}-p\right),
$$

provided that $p \neq x_{i}$. This definition is quite natural, and at least when $\alpha=-\pi$ has some nice properties: 


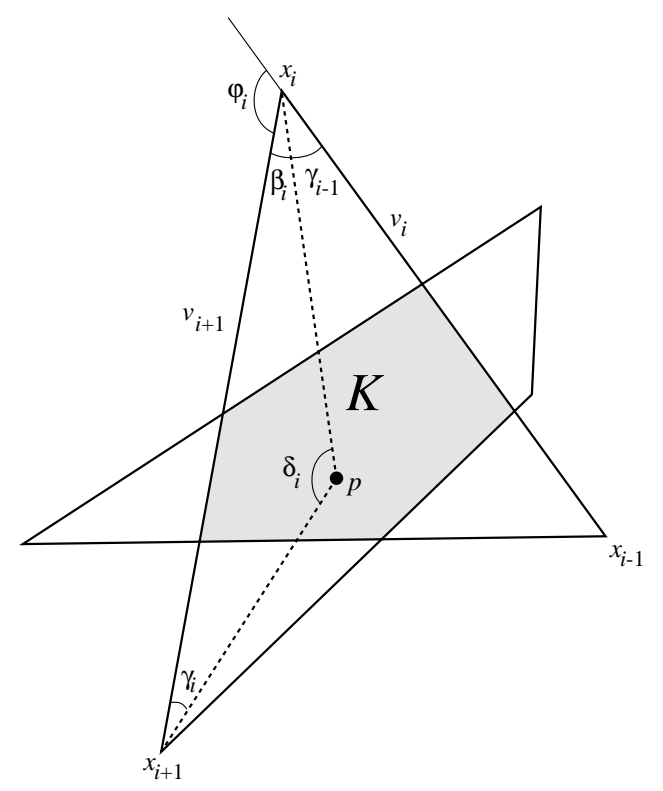

Figure 1: A polygon $\mathcal{P}_{6}$ and the turning angle $\varphi$. The angle subtended by the side $v_{i+1}$ is $\delta_{i}=\Phi_{\alpha}\left(x_{i}-p, x_{i+1}-p\right)$. The polygon is star convex, with kernel $K$.

a) Since $\mathcal{P}_{n}$ is closed, $n \rho_{p}$ is an integer. Moreover, since $\alpha<\Phi_{\alpha} \leq \alpha+2 \pi$,

$$
\frac{\alpha}{2 \pi}<\rho_{p}\left(\mathcal{P}_{n}, \alpha\right) \leq 1+\frac{\alpha}{2 \pi} .
$$

b) $\rho_{p}\left(\mathcal{P}_{n},-\pi\right)$ is invariant under deformations of $\mathcal{P}_{n}$, and motions of $p$ so long as $p$ does not cross a side of $\mathcal{P}_{n}$. This follows from the fact that $\Phi_{\alpha}$ is continuous until it reaches the value $\alpha$ or $\alpha+2 \pi$.

c) $\rho_{p}\left(\mathcal{P}_{n},-\pi\right)$ changes by $\pm \frac{1}{n}$ if $p$ crosses a side $\left[x_{i}, x_{i+1}\right]$ of $\mathcal{P}_{n}$, depending on the change of orientation of the triangle $\left[p, x_{i}, x_{i+1}\right]$.

d) If $p$ is outside the polygon then $\rho_{p}\left(\mathcal{P}_{n},-\pi\right)=0$ (A point $p$ is "outside" of $\mathcal{P}_{n}$ if $p$ can be moved to infinity without crossing the polygon).

The disadvantage of this notion of rotation number is its dependence on the position of the center point, $p$.

A rotation number that is independent of a choice of center is the "selfrotation number." To obtain this, define the "turning angle" of two successive 
sides of the polygon by

$$
\varphi_{i}=\Phi_{\alpha}\left(v_{i}, v_{i+1}\right),
$$

see Fig. 1. We define the self-rotation number of $\mathcal{P}_{n}$ to be the average turning angle:

$$
\rho_{s}\left(\mathcal{P}_{n}, \alpha\right)=\frac{1}{2 \pi n} \sum_{i=0}^{n-1} \varphi_{i},
$$

where $v_{n} \equiv v_{0}$.

For example, a "two polygon" has two sides $v_{0}=-v_{1}$. If the angle $\pi \in(\alpha, \alpha+2 \pi]$, then both $\Phi_{\alpha}\left(v_{0}, v_{1}\right)=\Phi_{\alpha}\left(v_{1}, v_{0}\right)=\pi$, and so $\rho_{s}=\frac{1}{2}$. More generally,

$$
\rho_{s}\left(\mathcal{P}_{2}, \alpha\right)=\frac{1}{2}+\left\lfloor\frac{\alpha}{2 \pi}+\frac{1}{2}\right\rfloor .
$$

where \lfloor\rfloor indicates the floor function.

When $\alpha=-\pi$, the rotation number of a triangle is $\pm \frac{1}{3}$ depending on whether it is oriented positively or negatively, respectively. However, for other values of $\alpha$, the situation is not quite so simple. For example if $\alpha=\frac{\pi}{2}$, then the rotation number of a positively oriented obtuse triangle is $\frac{2}{3}$, while that of a positively oriented acute triangle is $\frac{1}{3}$. We show some examples of 4 and 5 sided polygons and their rotation numbers in Fig. 2.

The self-rotation number has the following simple properties:

a) $\rho_{s}$ is independent of the position of the polygon in the plane, its orientation, and its scale.

b) Since $\mathcal{P}_{n}$ is closed, $n \rho_{s}$ is an integer. Moreover $\rho_{s}$ has the range (4).

c) The rotation number $\rho_{s}$ is constant under deformations of $\mathcal{P}_{n}$ providing adjacent sides do not have angle $\alpha$ : If $\alpha=-\pi$ this means that adjacent sides cannot cross.

The self-rotation number and the rotation number with respect to a point can be the same for certain polygons and an appropriate choice of $p$. For example, it can be easily seen that for any convex polygon, $\rho_{s}\left(\mathcal{P}_{n},-\pi\right)=$ $\rho_{p}\left(\mathcal{P}_{n},-\pi\right)$ when $p$ is in the interior of $\mathcal{P}_{n} .{ }^{1}$ More generally, consider the

\footnotetext{
${ }^{1}$ However, this result is not necessarily true for other choices of $\alpha$.
} 

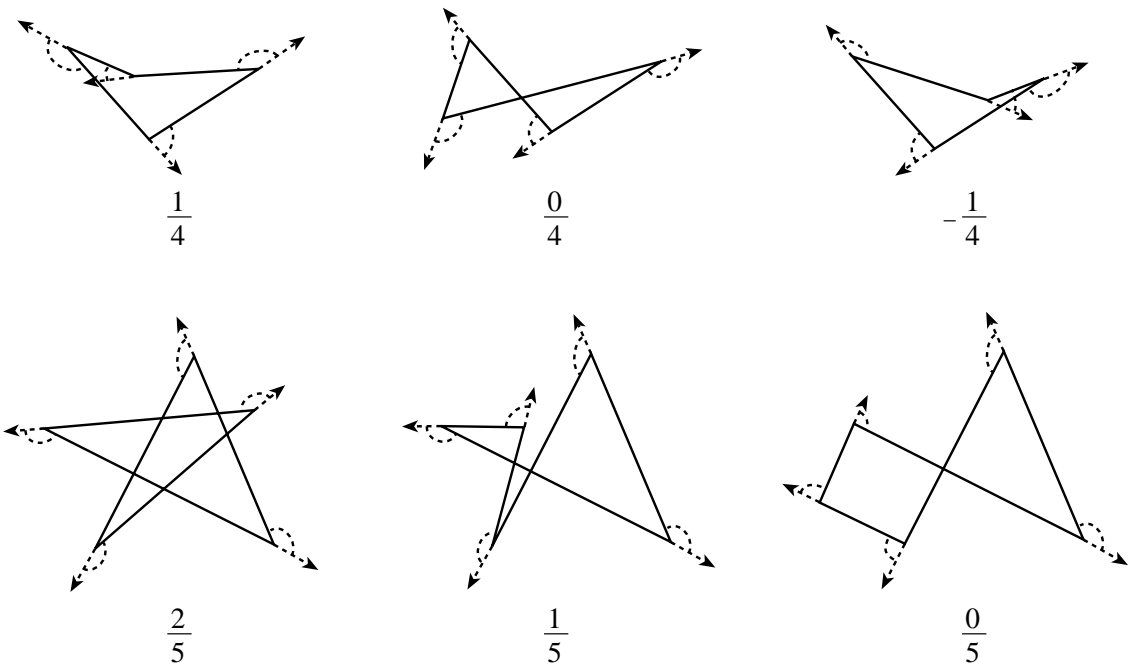

Figure 2: Self-rotation numbers for some simple polygons with $\alpha=-\pi$. If instead $\alpha=-\frac{3 \pi}{2}$ as in $\S 5.3$ then the rotation numbers become $-\frac{1}{4},-\frac{2}{4},-\frac{1}{4}$ and $-\frac{3}{5},-\frac{3}{5},-\frac{2}{5}$ respectively.

class of star-convex polygons:

Definition 1 (Open Star-Convex). A set $S$ is called open star-convex if there exists an open subset $K$ of $S$, the "kernel," such that for each $x \in K$, and every $y \in S$ the line segment connecting $x$ to $y$ lies entirely in $S$.

Star-convexity is typically defined without the requirement that the kernel be open, however, we need this property below. The kernel can be obtained as the intersection of the cones from all of the vertices of the polygon. According to this definition the $1^{\text {st }}, 3^{\text {rd }}$ and $4^{\text {th }}$ polygons in Fig. 2 are open star-convex. The $2^{\text {nd }}$ and $6^{\text {th }}$ polygons are star-convex, but their kernels are single points.

Lemma 1. Suppose $\mathcal{P}_{n}$ is an open star-convex polygon. Then for $\alpha=-\pi$ and each $p$ in the kernel of $\mathcal{P}_{n}$, the rotation number relative to $p$ equals the self-rotation number.

Proof. When $p \in K$, each of the triangles $T_{i}=\left[p, x_{i}, x_{i+1}\right]$ has the same orientation, because $p$ is in the interior of each of the cones from the vertices. Suppose that the triangles are positively oriented. The interior angle of $T_{i}$ at 


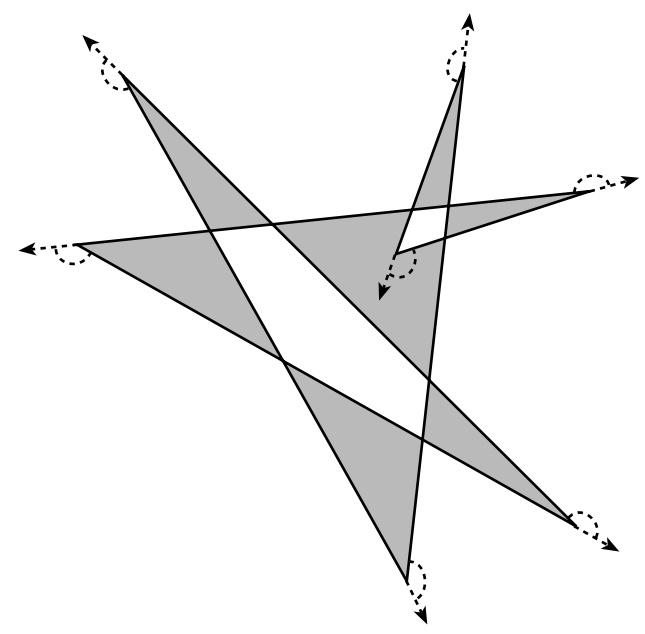

Figure 3: Polygon with rotation number $\rho_{s}\left(\mathcal{P}_{7},-\pi\right)=3 / 7$ for which $\rho_{p}\left(\mathcal{P}_{7},-\pi\right)<$ $3 / 7$ for every reference point $p$.

$p$ is $\delta_{i} \equiv \Phi_{-\pi}\left(x_{i}-p, x_{i+1}-p\right)$, and these angles are positive by assumption, see Fig. 1. Denote the interior angle of $T_{i}$ at $x_{i}$ by $\beta_{i}$ and that at $x_{i+1}$ by $\gamma_{i}$, so that $\delta_{i}+\beta_{i}+\gamma_{i}=\pi$. Note that the turning angles are also positive, and that $\Phi_{-\pi}\left(v_{i}, v_{i+1}\right)+\beta_{i}+\gamma_{i-1}=\pi$. Thus we have

$$
\begin{aligned}
\rho_{p}\left(\mathcal{P}_{n},-\pi\right) & =\sum_{i=0}^{n-1} \delta_{i}=\sum_{i=0}^{n-1}\left(\pi-\beta_{i}-\gamma_{i}\right)=\sum_{i=0}^{n-1}\left(\pi-\beta_{i}-\gamma_{i-1}\right) \\
& =\sum_{i=0}^{n-1} \Phi_{-\pi}\left(v_{i}, v_{i+1}\right)=\rho_{s}\left(\mathcal{P}_{n},-\pi\right) .
\end{aligned}
$$

The case when the polygon is negatively ordered follows similarly.

Though this lemma shows that the two rotation numbers agree for sufficiently nice polygons, there are polygons with more than six sides for which $\rho_{s}\left(\mathcal{P}_{n},-\pi\right) \neq \rho_{p}\left(\mathcal{P}_{n},-\pi\right)$ for any choice of $p$; see Fig. 3 for an example. 


\section{Self-Rotation Number for Maps}

The "self-rotation" number of Peckham [4] is defined for orbits of any homeomorphism $f$ on $\mathbb{R}^{2}$. We start by choosing distinct points $x$ and $y$ in $\mathbb{R}^{2}$, and letting:

$$
r(x, y)=\|y-x\|_{2}, \quad \theta(x, y)=\frac{y-x}{r},
$$

see Fig. 4. Here we use the natural embedding of $S$ into $\mathbb{R}^{2}$ so that $\theta \in S$ is represented by a unit vector in the plane. We can now extend $f$ to a map on pairs of points, and write the result in terms of $r$ and $\theta$ :

$$
r^{\prime}(x, y)=r(f(x), f(y)) \quad, \quad \theta^{\prime}(x, y)=\theta(f(x), f(y)) .
$$

Thus $f$ induces a map $F$ defined on $\mathbb{R}^{+} \times S \times \mathbb{R}^{2}$ given by

$$
\left(r^{\prime}, \theta^{\prime}, x^{\prime}\right)=F(r, \theta, x)
$$

where the vector $y-x$ is expressed as $(r, \theta, x) \in \mathbb{R}^{+} \times S \times \mathbb{R}^{2}$ using (6).

Since the space $\mathbb{R}^{+} \times S \times \mathbb{R}^{2}$ has a single noncontractible loop, we can construct a lift $\tilde{F}$ which is a map on $\mathbb{R}^{+} \times \mathbb{R} \times \mathbb{R}^{2}$. Here we denote the angle coordinate by $\tilde{\theta} \in \mathbb{R}$, and let $\tilde{\theta}^{\prime}=\tilde{F}_{2}(r, \tilde{\theta}, x)$. To construct the lift, select a base point $(x, y)$ and choose particular values for $\left(\tilde{\theta}, \tilde{\theta}^{\prime}\right)$ equivalent to the unit vectors $\left(\theta, \theta^{\prime}\right)$. The values elsewhere are obtained by continuity. For example, a lift could be constructed along an orbit by choosing any curve $\mathcal{C}=\{(x(t), y(t)): 0 \leq t \leq 1\}$ that connects the base point $\mathcal{C}(0)=(x, y)$ to $\mathcal{C}(1)=(f(x), f(y))$ so that $\tilde{\theta}(t)=\tilde{\theta}(x(t), y(t))$ is continuous and $\tilde{\theta}^{\prime}=\tilde{\theta}(1)$. Then the image of this curve, $F(\mathcal{C})$ continuously connects $\tilde{\theta}^{\prime}$ to the next angle $\tilde{\theta}^{\prime \prime}$, see Fig. 4.

The self-rotation number is the rotation rate of a point $x$ about itself. To obtain this, we restrict the map $\tilde{F}$ to the graph $y=f(x)$. The restriction is well defined provided that $x$ is not a fixed point of $f$. The rotation number is defined as the average increase in $\tilde{\theta}$ per iteration, just as for the circle map (1):

$$
\rho_{s}(x)=\lim _{t \rightarrow \infty} \frac{1}{2 \pi t}\left[\tilde{F}_{2}^{t}(r(x, f(x)), \tilde{\theta}(x, f(x)), x)-\tilde{\theta}(x, f(x))\right],
$$




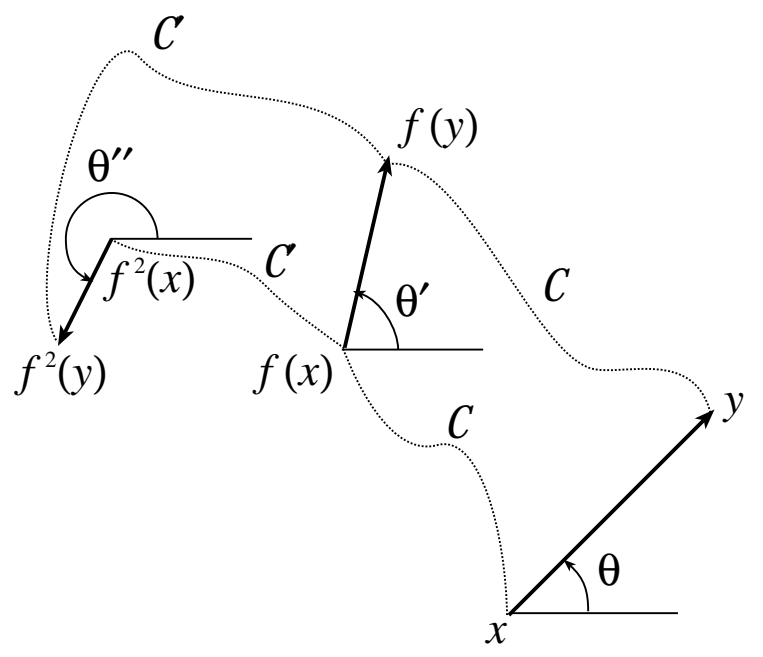

Figure 4: Sketch of the polar coordinates and the lift to define the self-rotation number.

providing this limit exists. Just as for the rotation number (1), $\rho_{s} \bmod 1$ is independent of the choice of lift. The self-rotation number has several nice properties [4]:

a) If $f$ has an invariant circle $S \in \mathbb{R}^{2}$, then for an orbit on the circle the self-rotation number is the circle map rotation number.

b) $\rho_{s}$ can be extended to a fixed point of $f$ by defining the rotation number of $x^{*}$ to be $\lim _{x \rightarrow x^{*}} \rho_{s}(x)$.

c) The self-rotation number of a periodic orbit cannot change under continuous parameter changes, since it is a rational number.

The construction of $\tilde{F}$ may be difficult to do explicitly. For example, consider the Hénon map,

$$
f\left(x_{1}, x_{2}\right)=\left(x_{2}-k+x_{1}^{2},-b x_{1}\right) .
$$

An example of the unit vector field $\theta(x, f(x))$ for this map in shown in Fig. 5 . The map has two fixed points when $4 k+(1+b)^{2}>0$, one has Poincaré index -1 , the other and has Poincaré index +1 . The change in $\tilde{\theta}$ on a loop 
encircling a single fixed point is $2 \pi$ times the Poincaré index. Thus the angle $\tilde{\theta}(x, f(x))$ at some general point $x$ will depend upon the number of times the path encircles the fixed points. However, the angle $\tilde{\theta}^{\prime}$ will similarly change with the path in such a way that the difference $\tilde{\theta}^{\prime}-\tilde{\theta}$ stays fixed. This is the only quantity that matters for the computation of $\rho_{s}$.

\section{Turning Angle}

An alternative formulation of the self-rotation number (7) is obtained by noting that it is the average rate of turning of the vector $f(x)-x$ along the orbit. Thus define the "turning angle" $\varphi: \mathbb{R}^{2} \rightarrow S$

$$
\varphi(x)=\tilde{\theta}^{\prime}\left(f^{-1}(x), x\right)-\tilde{\theta}\left(f^{-1}(x), x\right)
$$

see Fig. 6. We can think of $\varphi$ either as a unit vector field or as an angle, as is convenient. Using the turning angle, the self-rotation number can be written

$$
\rho_{s}(x)=\lim _{t \rightarrow \infty} \frac{1}{2 \pi t} \sum_{i=1}^{t} \varphi\left(f^{i}(x)\right) .
$$

We show an example of the unit vector field $\varphi$ for the Hénon map in Fig. 7. It surprised us that the vector $\varphi$ avoids an interval on $S$. In fact it appears that $\varphi$ maps $\mathbb{R}^{2}$ into some open subset of the circle, $I \subset S$. We will show that this is true in $\S 5.3$ when $b>0$. In this case, for any angle $\alpha \in S \backslash I$, the function $\varphi: \mathbb{R}^{2} \rightarrow(\alpha, \alpha+2 \pi]$ is continuous, and we say that the map $f$ avoids the angle $\alpha$.

Definition 2 (Avoids an Angle). A homeomorphism $f$ of the plane avoids an angle $\alpha$ if the turning angle $\varphi$ in (9) has a range that does not include $\alpha$.

When a map avoids an angle, the turning angle $\varphi$ can be obtained using $\Phi_{\alpha},(3)$, where successive iterates form the vectors $v_{1}$ and $v_{2}$,

$$
\varphi(x)=\Phi_{\alpha}\left(x-f^{-1}(x), f(x)-x\right) .
$$

The self-rotation number computed using the turning angle inherits the invariance property of the more general definition: 


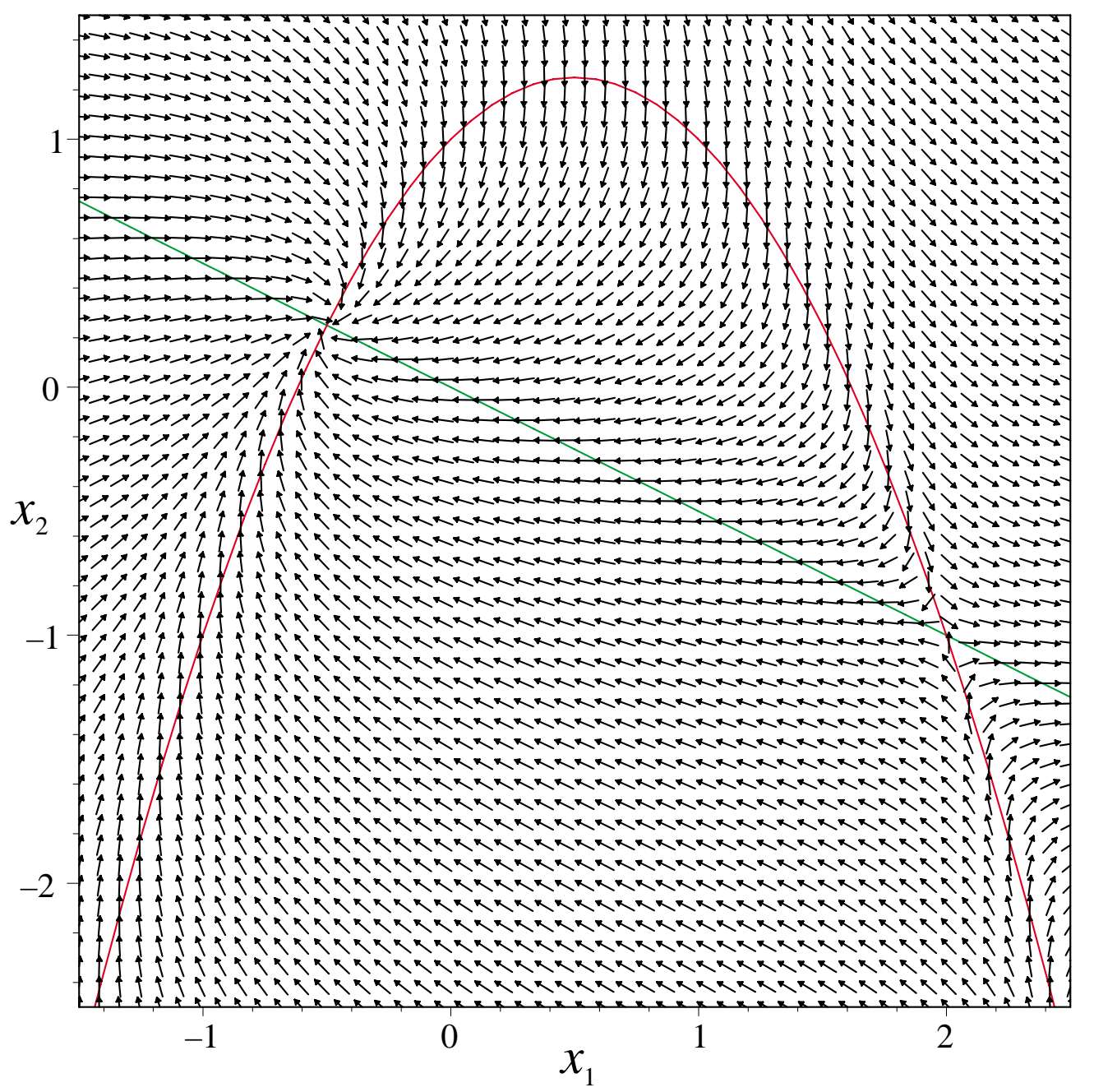

Figure 5: The vector field $\theta(x, f(x))$ for the Hénon map (8) with $k=1.0$ and $b=0.5$. The curves $x_{2}=-x_{1}$ and $x_{2}=x_{1}+k-x_{1}^{2}$ correspond to the isoclines where the vectorfield is horizontal or vertical, respectively 


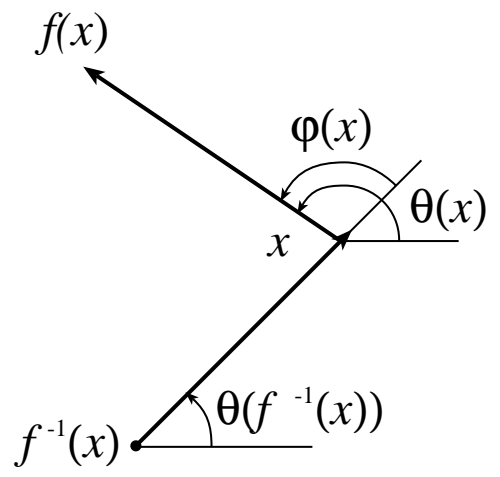

Figure 6: Sketch of the turning angle, defined by (9).

Theorem 2. If a one parameter family of maps of the plane $f_{\lambda}$ avoids the angle $\alpha(\lambda)$ then the self-rotation number $\rho_{s}(x)$ computed using (11) is an invariant under continuation for periodic orbits of $f_{\lambda}$.

Proof. A slight change in $\lambda$ induces a slight change in the periodic orbit. But the sum of the angles $\Phi_{\alpha(\lambda)}$ around the periodic orbit can only change if the turning angle reaches $\alpha(\lambda)$. Since this angle is avoided by the map $\rho_{s}$ is a constant.

A necessary condition for $\varphi,(9)$, to avoid an angle is that its restriction to any circle in phase space $\mathbb{R}^{2}$ (not containing any fixed points) is a circle map with degree zero (Recall that a map from $S$ to $S$ has degree $k$ if the image wraps around the circle $k$ times). Equivalently, if there is a circle for which $\varphi$ has nonzero degree, the map does not avoid an angle. Since the degree of a map is a homotopy invariant, any circle in the plane can be arbitrarily deformed as long as it does not cross fixed points, where $\varphi$ is not continuous. Since any circle that does not contain fixed points can be deformed to a point, $\varphi$ necessarily has degree zero on such circles. Similarly, we can deform a circle that encloses a single fixed point to an arbitrarily small circle, so that the linearization at the fixed point determines the degree. As we will see in $\S 4.1$, the degree of $\varphi$ on such a circle is zero if $f$ is orientation preserving, and is \pm 2 otherwise.

Even when $\varphi$ has degree zero on every circle, $f$ may not avoid an angle. 


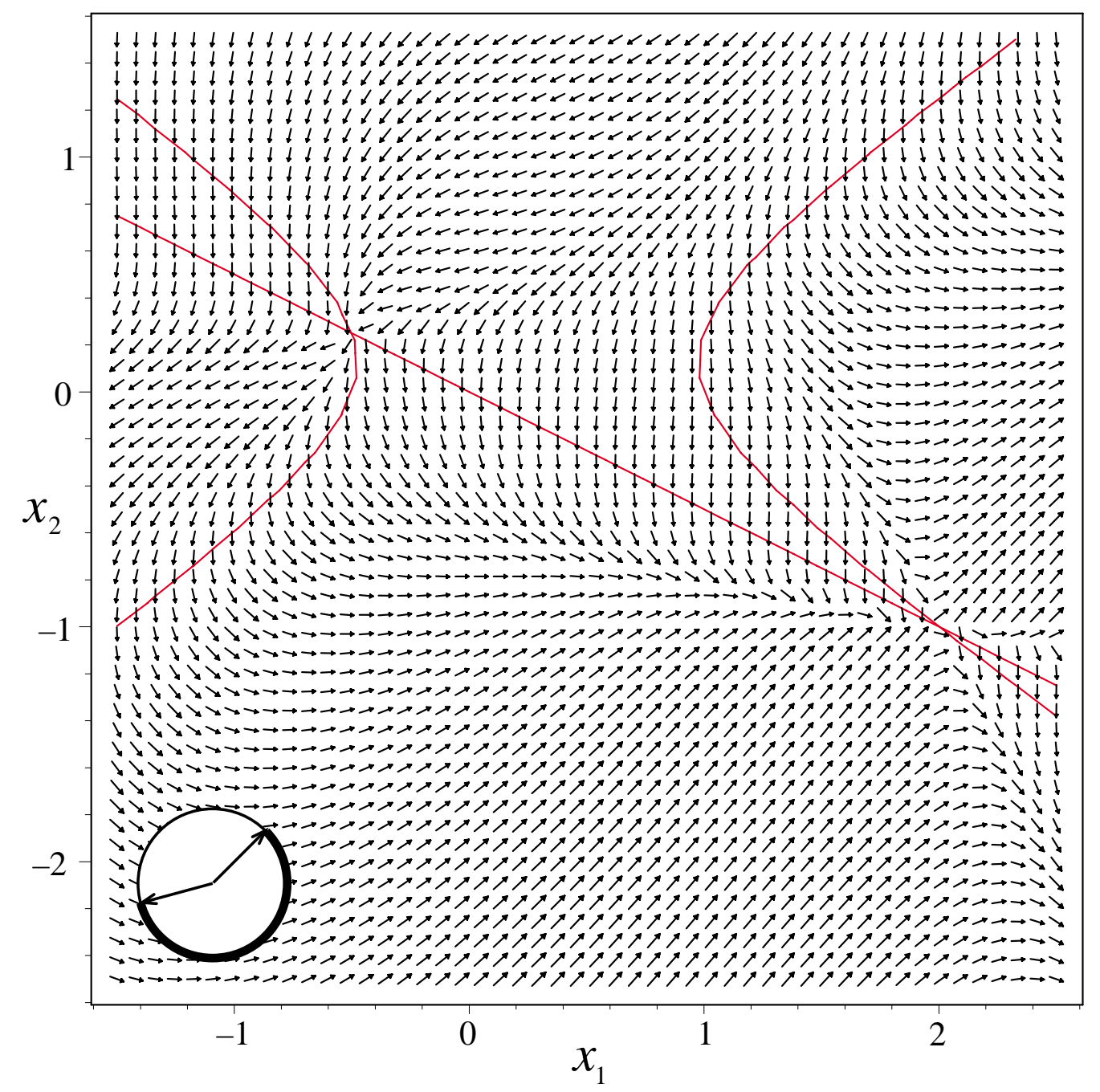

Figure 7: The turning angle vector field $\varphi(x)$ for the Hénon map (8) with $k=1$ and $b=0.5$. The turning vector is vertical on the line and hyperbola shown. Also shown, at the lower left, is the range of the turning angle. 
For example, $\varphi$ need not be monotone and its range might be the entire circle. Even when the range on a given circle avoids some angle $\alpha_{0}$, the range on other circles may not avoid this particular angle, since the avoided angle could change with deformation of the circle.

We can compute the global maximum and minimum of $\varphi$ to help determine whether an angle is avoided. The extrema are attained either at smooth critical points of $\varphi$, at the fixed points of $f$, or at infinity. We will find the extrema for fixed points in the next subsection. The critical points of $\varphi$ are given by

Lemma 3. The critical points of $\varphi(x)$ occur when the two conditions

$$
\frac{\partial w}{\partial x_{1}} \times w=0, \quad \frac{\partial w}{\partial x_{2}} \times w=0,
$$

are fulfilled. Here $w=\left(v_{1} \cdot v_{2}, v_{1} \times v_{2}\right)$ and $v_{1}=x-f^{-1}(x), v_{2}=f(x)-x$.

Proof. By (3) and (11), $\varphi=\arctan _{\alpha}\left(v_{1} \times v_{2}, v_{1} \cdot v_{2}\right)$. Requiring the derivatives with respect to both components of $x$ to be zero gives the above result.

\subsection{Linear Maps}

The turning angle (9) is singular at the fixed points of the map, because there both $v_{1}$ and $v_{2}$ are zero. However, if $f$ is a diffeomorphism, then the turning angle has a limit on any ray approaching the fixed point. To calculate this limit it is enough to consider the linearization of the map at the fixed point. Denote the linear map by the matrix $D f\left(x^{*}\right)=L$. Since $f$ is a diffeomorphism, $L$ is nonsingular. For the moment, we also assume that $L$ has a unique fixed point, which is equivalent to the matrix $L-I$ being nonsingular.

To compute the turning angle for $L$, we use the vectors $v_{1}=\left(I-L^{-1}\right) x$ and $v_{2}=(L-I) x=L v_{1}$. Since the map is linear, the magnitude of $x$ is unimportant, so we can assume that $x=(\cos \psi, \sin \psi)$ is a unit vector. Then $v_{1}$ can be represented in the polar coordinates (6) to give the angle $\theta(\psi)$. When the fixed point is unique, the image of the unit circle under the map 
$I-L^{-1}$ is an ellipse. ${ }^{2}$ In this case, the map from $x$ to $v_{1}$ is one-to-one and the orientation of the rotation on the ellipse is determined by the sign of $\operatorname{det}\left(I-L^{-1}\right)$; that is, the circle map $\theta(\psi)$ has degree $\operatorname{sign}\left(\operatorname{det}\left(I-L^{-1}\right)\right)$. We show an example in Fig. 8. Similarly, the map from $\psi$ to $\theta^{\prime}$, which is induced by the nonsingular matrix $L-I$ is a degree $\operatorname{sign}(\operatorname{det}(L-I))$ circle map. Now, since $\operatorname{det}(L-I)=\operatorname{det}(L) \operatorname{det}\left(I-L^{-1}\right)$, when $L$ is orientation preserving these two maps have the same degree. This implies that the turning angle, $\varphi=\tilde{\theta}^{\prime}-\tilde{\theta}$, is a degree zero circle map. However, if $L$ is orientation reversing, then the two maps $\theta^{\prime}(\psi)$ and $\theta(\psi)$ have opposite orientations. This implies that $\varphi$ has degree $2 \operatorname{sign}(\operatorname{det}(L-I))$.

We conclude that it is possible for a linear map to avoid an angle only if it is orientation preserving. In fact we can show

Lemma 4. Suppose $L$ is an orientation preserving, nonsingular, linear map. Then $L$ avoids an angle $\alpha$.

Proof. We first assume that $L-I$ is nonsingular, so that without loss of generality, we can assume that $v_{1}$ points in a general direction.

Since the turning angle is the angle between the vectors $v_{1}$ and $L v_{1}$, it is independent of the magnitude of $v_{1}$. Therefore, we can write $v_{1}=$ $(\cos \theta, \sin \theta)$. Whenever $L$ is nonsingular, it maps the circle $v_{1}=(\cos \theta, \sin \theta)$ one-to-one onto an ellipse. Thus the map from $\tilde{\theta}$ to $\tilde{\theta}^{\prime}$ is monotone increasing. Since $\tilde{\theta}^{\prime}=\tilde{\theta}+\varphi$, then $\frac{d \varphi}{d \theta}>-1$. An elementary application of the mean value theorem then implies that $\varphi$ has range less than $2 \pi$. For if the range of $\varphi$ were at least $2 \pi$, then there would be points $0 \leq \theta_{0}, \theta_{1}<2 \pi$ for which $\varphi\left(\theta_{1}\right)-\varphi\left(\theta_{0}\right)=2 \pi$. Either $0<\theta_{0}-\theta_{1}<2 \pi$, or $0<\theta_{0}+2 \pi-\theta_{1}<2 \pi$. In either case, since $\varphi$ is periodic, the mean value theorem implies there must be a point for which $\frac{d \varphi}{d \theta}<-1$.

If $L-I$ is singular, then there are three possibilities. If $L$ is the identity matrix, every point is fixed and the turning angle is not defined. If $L$ has

\footnotetext{
${ }^{2}$ For example, let $M \equiv I-L^{-1}=\left(\begin{array}{ll}a & b \\ c & d\end{array}\right)$. The image of the unit circle under $M$ is the ellipse

$$
\left(c^{2}+d^{2}\right) x^{2}-2(a c+b d) x y+\left(a^{2}+b^{2}\right) y^{2}=\operatorname{det}(M)^{2} .
$$
}

Thus the ellipse has nonzero radii whenever $\operatorname{det}(M) \neq 0$. 
a)

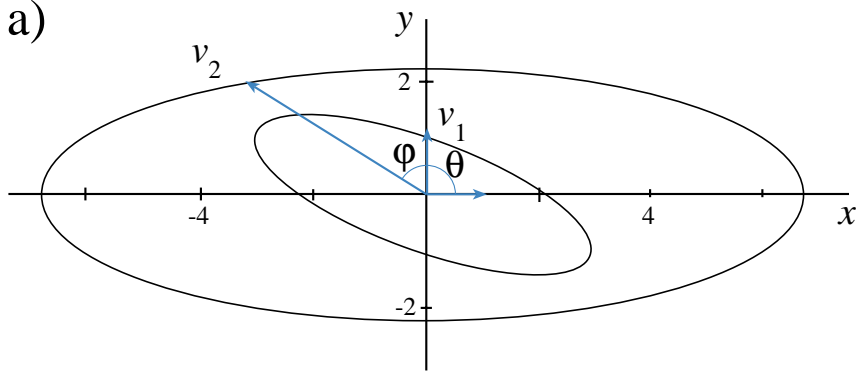

b)

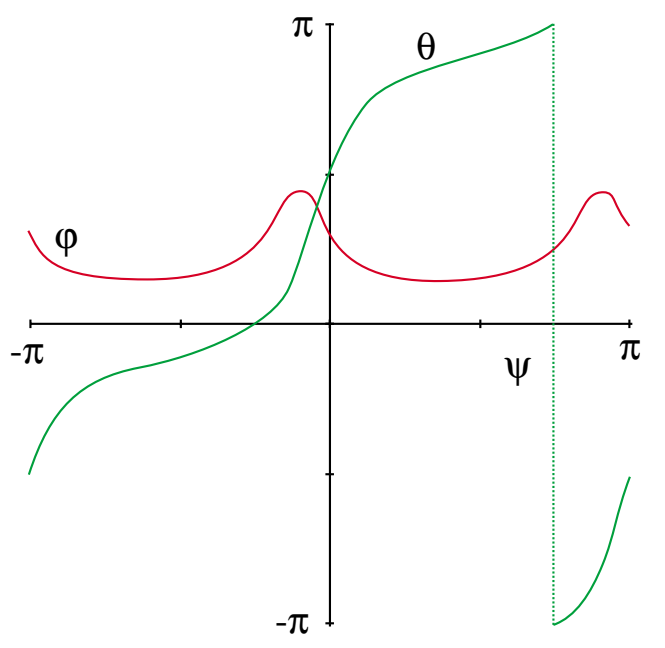

Figure 8: The turning angle for a linear map $L=\left(\begin{array}{cc}1 & -3 \\ 1 & 2\end{array}\right)$. In (a), the ellipses are the curves traced out by vectors $v_{1}$ and $v_{2}$ as $x$ encircles the origin. The curves in (b) are $\theta(\psi)$ and $\varphi(\psi)$. 
a single eigenvalue 1 , then there is a line of fixed points along its eigenvector. For any point not on this line, $v_{1}$ points in the direction of the second eigenvector and so does $v_{2}$, so the turning angle is zero. If $L$ has a double eigenvalue 1 and is not the identity then it has nontrivial Jordan form. Now for any point that is not fixed, $v_{1}=v_{2}$ is the single eigenvector, so that $\varphi=0$. Note that a small perturbation can discontinuously change the range of $\varphi$ when $L-I$ is singular.

Therefore in all cases, $\varphi$ has range less than $2 \pi$, and $L$ avoids an angle.

Letting $L=\left(\begin{array}{ll}a & b \\ c & d\end{array}\right)$, we can write

$$
\begin{aligned}
\varphi(x) & =\arctan _{\alpha}\left(v_{1} \times v_{2}, v_{1} \cdot v_{2}\right), \\
v_{1} \times v_{2} & =c \cos ^{2} \theta+(d-a) \cos \theta \sin \theta-b \sin ^{2} \theta, \\
v_{1} \cdot v_{2} & =a \cos ^{2} \theta+(b+c) \cos \theta \sin \theta+d \sin ^{2} \theta,
\end{aligned}
$$

for a suitable choice of $\alpha$. To determine the actual range of $\varphi$, we find its critical points.

Lemma 5. Suppose $L=\left(\begin{array}{ll}a & b \\ c & d\end{array}\right)$ is the matrix of an orientation preserving, nonsingular, linear map with a unique fixed point. Then the maximum and minimum values of the turning angle are given by

$$
\tan \varphi_{ \pm}=\frac{\tau(c-b) \pm 2 w}{4 \delta-(c-b)^{2}}, \quad w=\sqrt{\delta\left(\tau^{2}-4 \delta+(c-b)^{2}\right)}
$$

respectively, where $\tau$ and $\delta$ are the trace and determinant of $L$.

The critical values are invariant under scaling $L$, which they should be, because for a linear map, $\varphi$ is constant on rays emerging from the origin. They are also invariant under rotations, i.e., the critical values are unchanged by the similarity transformation $R^{t} L R$ for any rotation matrix $R$. The extremal values are not coordinate independent: they do depend on the antisymmetry, $c-b$, of $L$. Finally, $w$ is real whenever $L$ is orientation preserving, as the factor under the square root can be written $\delta\left((a-d)^{2}+(b+c)^{2}\right)$, which is nonnegative when $\delta>0$. This also shows that when $\delta<0, \varphi$ has no real critical points. 
Proof. As before, $\varphi$ is independent of the magnitude of $v_{1}$, so we can set $v_{1}=(\cos \theta, \sin \theta)$. Elementary calculations using (12) give the critical points

$$
\tan \theta_{ \pm}=\frac{a b+c d \mp w}{\delta-b^{2}-d^{2}}
$$

providing $L$ is orientation preserving - for then $w$ is real. Note that there are actually 4 critical points, since if $\theta$ is a critical point, so is $\theta+\pi$. The second derivative of $\varphi$ at the critical point is $\mp \frac{2 w}{\delta}$, showing that the upper sign is a maximum and the lower sign a minimum. Substituting the critical points into the expression for $\varphi$ gives the promised critical values.

Since (13) is an equation only for the tangent of the extrema, one must choose an appropriate branch to determine the actual range of $\varphi$. To do this, first find one point in the range of $\varphi$; for example, (12) implies that $\varphi((1,0))=\arctan (c, a)$. Depending upon the sign of the denominator of (13), unique branches can be chosen so that $\varphi_{-} \leq \varphi((1,0)) \leq \varphi_{+}$. For the example in Fig. 8, a,c >0, and the denominator is positive, so that $-\frac{\pi}{2}<\varphi_{-}<\varphi_{+}<\frac{\pi}{2}$.

\section{Applications}

Here we present some examples of maps that avoid an angle $\alpha$. In particular, we show that certain parameterized families of maps avoid a fixed angle. Once the value of $\alpha$ is known, we can compute self-rotation numbers for periodic orbits at any parameter value, and we know the rotation number cannot change. In particular, we know that two period $n$ orbits with different self-rotation numbers cannot collide. Thus we obtain restrictions on the bifurcations that can occur.

\subsection{Nearly Linear Maps}

Since a linear, orientation preserving map with a unique fixed point avoids some angle $\alpha$, then any map nearby will avoid the same angle. For example, consider the diagonal map, $L=\left(\begin{array}{ll}a & 0 \\ 0 & d\end{array}\right)$, and suppose that $0<a<d$. If $a=1$ 
or $d=1$, then $\varphi(x) \equiv 0$. Otherwise, we have

$$
v_{1} \times v_{2}=(d-a) \sin (2 \theta), \quad v_{1} \cdot v_{2}=a+d-(d-a) \cos (2 \theta) .
$$

Note that the dot product is always positive. In fact the turning angle is always in the interval

$$
|\varphi|<\arctan \left(\frac{d-a}{\sqrt{a d}}\right)<\frac{\pi}{2} .
$$

Therefore the angle $\alpha=-\pi$ is avoided.

Thus, any perturbation that does not twist the vectors too much also will avoid the angle $-\pi$. For example if

$$
f(x)=L x+\epsilon g(x)
$$

and $|g(x)|<|x|$, then the vectors $v_{i}$ will be changed only by $\mathcal{O}(\epsilon)$, and for small enough $\epsilon$, the map will still avoid $-\pi$.

\subsection{Birkhoff Normal Form}

The Birkhoff normal form for a map with an elliptic fixed point is

$$
r^{\prime}=r, \psi^{\prime}=\psi+\Omega(r)
$$

where $(r, \psi)$ are polar coordinates and $\Omega$ is the rotation number. For this map, the turning angle is precisely $\Omega(r)$. If the range of $\Omega$ is less than $2 \pi$, then the map avoids an angle, and we can use our formulation to compute the self-rotation number. For example, suppose that $\Omega(r)=\omega_{0}+a \tanh (r)$, then the range of the turning angle is $\left[\omega_{0}, \omega_{0}+a\right)$, so for any $0<a<2 \pi$, the map avoids an angle.

A map that is nearby, e.g.,

$$
r^{\prime}=r+\epsilon g_{1}(r, \psi), \psi^{\prime}=\psi+\Omega(r)+\epsilon g_{2}(r, \psi),
$$

where $\left|g_{1}\right|<r$, and $\left|g_{2}\right|<1$, will also avoid an angle if $\epsilon$ is small enough. 


\subsection{Hénon Map}

Our motivation for developing the turning angle was a detailed study of the periodic orbits of the Hénon map [5]. Recall that this map is given by (8)

$$
f(x, y)=\left(y-k+x^{2},-b x\right),
$$

where we now use $(x, y)$ for the coordinates to avoid excessive subscripting. Since $\operatorname{det} D f=b$, according to the linear map results, we cannot expect the Hénon map to avoid any angle when $b<0$, since then it is orientation reversing. However, otherwise, we can show

Theorem 6. The orientation preserving Hénon map $(b>0)$ avoids the angle $\alpha=-3 \pi / 2$.

Proof: The turning angle is given by (11), where the two vectors are $v_{1}=(x, y)-f^{-1}(x, y)$ and $v_{2}=f(x, y)-(x, y)$. In order to show that $\varphi \neq-3 \pi / 2$ we could compute the minimum and maximum values of $\varphi(x)$ as we did for the linear map in Lemma 5 and then show that $-3 \pi / 2$ is not in the interval between them. This would give the complete range of $\varphi(x)$ that is avoided, but here we only need to show that $\varphi(x)$ avoids $-3 \pi / 2$. Since

$$
\tan \varphi(x) \equiv \frac{v_{1} \times v_{2}}{v_{1} \cdot v_{2}}
$$

we must show that when the vectors are perpendicular the cross product is negative. Note that when the vectors are perpendicular, $v_{1} \cdot v_{2}=0$, and

$$
v_{1} \times\left. v_{2}\right|_{\left\{v_{1} \cdot v_{2}=0\right\}}= \pm\left|v_{1}\right|\left|v_{2}\right|,
$$

so we must show that only the - sign occurs. Generically the dot product will vanish on some set of curves. On these curves the vector $\varphi$ is vertical; we must show that it points down. According to (14), the cross product cannot change sign on one of these curves unless one (or both) of the two vectors $v_{i}=0$. If either vanishes, then the point $(x, y)$ is a fixed point, and both vectors vanish. However, the results of the previous section imply that the sign of the cross product cannot change if a curve goes smoothly through a fixed point, because the angle $\varphi$ for a linear map is constant on lines through the fixed point. Thus for smooth curves, $\left\{(x, y): v_{1} \cdot v_{2}=0\right\}$, we can evaluate 
the cross product at any point on the curve, and if it is negative at the point, it is negative everywhere on the curve.

Specifically for the Hénon map, the dot product is

$$
v_{1} \cdot v_{2}=\frac{1}{b^{2}}(y+b x)\left[b\left(x-x_{0}\right)^{2}-\left(y-y_{0}\right)^{2}+c\right],
$$

where $x_{0}=\frac{1-b}{2}, y_{0}=b x_{0}$, and $c=\frac{b}{4}(b-1)\left[(b-1)^{2}+4 k\right]$. Thus the dot product vanishes on three curves in the plane: the line $y=-b x$ and the two branches of the hyperbola defined by the equation in the brackets (recall Fig. 7). These curves cross at the fixed points of the map and each curve goes to infinity in the plane. It is particularly easy to evaluate the sign of the cross product near infinity on these curves. The cross product is

$$
v_{1} \times v_{2}=-\frac{1}{b}(y+b x)^{2}-\left(y-x-k+x^{2}\right)\left(y-x-k+\frac{y^{2}}{b^{2}}\right) .
$$

It is easy to see that on the line $y=-b x$, we have

$$
v_{1} \times\left. v_{2}\right|_{\{y=-b x\}}=-\left((1+b) x+k-x^{2}\right)^{2},
$$

which is obviously nonpositive, and vanishes only at the fixed points. The asymptotes of the hyperbola are the lines $y= \pm \sqrt{b} x$. On these lines near infinity, the cross product becomes

$$
v_{1} \times\left. v_{2}\right|_{\{y= \pm \sqrt{b} x\}} \rightarrow-\frac{x^{4}}{b}, \quad \text { as } x \rightarrow \infty .
$$

Thus we see that on each of the three curves, $v_{1} \times v_{2} \leq 0$ and so the angle $-3 \pi / 2$ is avoided.

Choosing $\alpha=-\frac{3 \pi}{2}$ gives a self-rotation number in the domain $\left(-\frac{3}{4}, \frac{1}{4}\right]$. In fact the predominant rotation for the Hénon map with our convention is clockwise, and so rotation numbers are negative. We will see in $\S 5.5$ that positive rotation numbers cannot occur for bounded orbits.

We show in Fig. 9 a period 5 orbit for three values of $k$ when $b=1$. This orbit is one of a pair created in a rotational bifurcation of the elliptic fixed point when $k=\frac{7+5 \sqrt{5}}{8} \approx 2.27254$, where the multipliers of the elliptic 


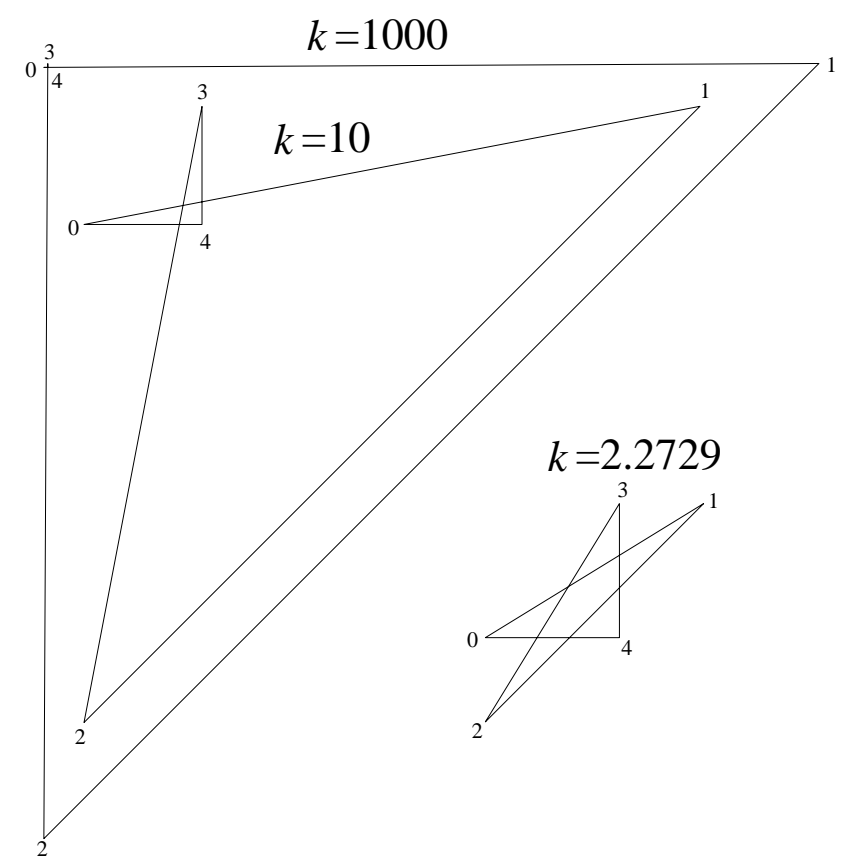

Figure 9: The period 5 orbit with symbol sequence $(----+)^{\infty}$ shown for 3 values of $k$ at $b=1$. The orbits are not shown to scale: the star should be about 3000 times smaller than the triangle. The labels in the figure indicate the order in which the motion occurs.

fixed point are $e^{ \pm 2 \pi i \frac{2}{5}}$. Since the motion is clockwise, the rotation number of the orbit should be $-\frac{2}{5}$. This is indeed what is obtained for the selfrotation number, as the orbit has the expected "star" shape, and all of the turning angles are near $-4 \pi / 5$ for $k$ near the bifurcation value. Near $k=2.5$, however, one vertex of the star moves across a side, though the orbit remains star-convex. As $k \rightarrow \infty$, three of the vertices approach each other, and the orbit limits to a triangle. Nevertheless, the self-rotation number remains $-\frac{2}{5}$, since none of the turning angles can cross the avoided $\alpha$. For this orbit the specific choice $\alpha=-\frac{3 \pi}{2}$ is not critical, and a naive choice of $\alpha=-\pi$ to compute the self-rotation number would give the correct value.

In Fig. 10, we show a period 6 orbit for four values of $k$ when $b=1$. This orbit is created in a saddle-center bifurcation at $k \approx 3.70166$. The self- 


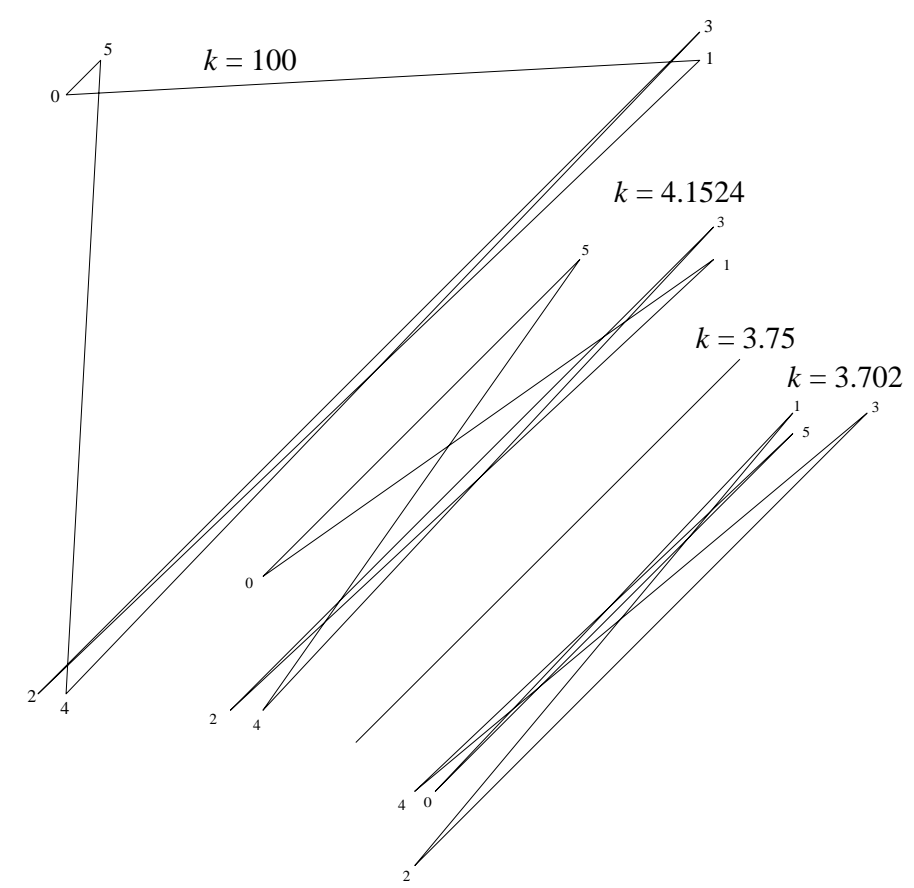

Figure 10: Period 6 orbit with symbol sequence $(--+-+-)^{\infty}$ shown for four values of $k$ for $b=1$. The orbits are not shown to scale. The labels in the figure indicate the order in which the motion occurs.

rotation number is easily seen to be $\rho_{s}=-\frac{3}{6}$. Since this orbit has the same rotation number as the period 2 orbit, it is possible for these orbits to collide; indeed this happens at $k=\frac{15}{4}$, where the multipliers of the period two orbit are $e^{ \pm 2 \pi i \frac{1}{3}}$. We can then identify this orbit as a tripled period two orbit. It is interesting that as $k$ passes through $\frac{15}{4}$, several of the turning angles pass through $-\pi$. Thus the choice of $\alpha=-\frac{3 \pi}{2}$ is important to compute the correct rotation number for this orbit. As $k \rightarrow \infty$, this orbit limits on the same triangle as the previous orbit.

Generally the self-rotation number can be used to obtain restrictions on the possible phase space configurations of an orbit, and on the possible bifurcations that it can undergo. We will investigate this in $§ 5.5$. 


\subsection{Generalized Hénon maps}

Maps that have the generalized Hénon form [6]

$$
f(x, y)=(y-k+p(x),-b x),
$$

for any function $p(x)$, may also avoid an angle when they are orientation preserving. The dot and cross products are given by

$$
\begin{aligned}
v_{1} \cdot v_{2} & =\frac{1}{b^{2}}(y+b x)[(1-b)(y-x-k)+p(x)-b p(-y / b)], \\
v_{1} \times v_{2} & =-\frac{1}{b}(y+b x)^{2}-(y-x-k+p(x))(y-x-k+p(-y / b)) .
\end{aligned}
$$

To show that the angle $-3 \pi / 2$ is avoided, we must again study the curves where $v_{1} \cdot v_{2}=0$. Our considerations of the previous section show that we need evaluate the cross product only at one point on each curve defined by the above equation. However, in this case, all of the curves need not extend to infinity. A simple case arises if one can show that a curve intersects a fixed point, $x^{*}$. For then we can linearize the map at the fixed point, and the formula for the dot product and cross product become

$$
\begin{aligned}
\left.v_{1} \cdot v_{2}\right|_{D f\left(x^{*}\right)} & =\xi\left[p^{\prime}\left(x^{*}\right) \xi+(1-b) \eta\right], \\
v_{1} \times\left. v_{2}\right|_{D f\left(x^{*}\right)} & =-b \xi^{2}-p^{\prime}\left(x^{*}\right) \xi \eta-\eta^{2},
\end{aligned}
$$

where $v_{1}=(\xi, \eta)$. Now the curves of vanishing dot product are the lines $\xi=0$, and $\eta=\frac{p^{\prime}\left(x^{*}\right)}{b-1} \xi$. Substitution of these values into the cross product leads to

$$
v_{1} \times v_{2}=\left\{\begin{array}{c}
-\eta^{2} \\
-b \xi^{2}\left(1+\frac{p^{\prime}(x)^{2}}{(b-1)^{2}}\right)
\end{array}\right.
$$

which in both cases is manifestly nonpositive.

Another simple situation arises if a curve of vanishing dot product approaches infinity. Suppose, for example that $p$ is unbounded and grows faster than linearly. Then if the dot product is to vanish near infinity, either $y=-b x$ or $p(x) \rightarrow b p(-y / b)$. For the first case it is easy to see that the cross product is nonpositive as before; for the second

$$
v_{1} \times v_{2} \rightarrow-\frac{1}{b} p(x)^{2}<0 \text { as } x, y \rightarrow \infty .
$$


Thus if the curves of vanishing dot product are not compact or intersect a fixed point, the map avoids $-3 \pi / 2$. We do not have a general demonstration that these curves have either of these two properties.

\subsection{Rotation Number near the Anti-Integrable Limit}

The Hénon map has an anti-integrable (AI) limit [7] corresponding to $k \rightarrow \infty$. This is most easily seen by defining a new parameter $\epsilon=\frac{1}{\sqrt{k}}$ and the new variables $z=\epsilon x$, and $w=\epsilon y$, so that the map (8) becomes

$$
\left(z^{\prime}, w^{\prime}\right)=f(z, w)=\left(\frac{1}{\epsilon}\left(z^{2}-1\right)+w,-b z\right)
$$

At the AI limit, $\epsilon=0$, there is no longer a dynamical system; instead the orbits become sequences $\left(z_{t}, w_{t}\right)=\left(s_{t},-b s_{t-1}\right)$, where the symbols $s_{t}= \pm 1$ change arbitrarily from step to step. We often use just the signs + and - to denote the symbols $s_{t}$. At the AI limit, the allowed states in the phase space $(z, w)$ correspond to the corners of the rectangle, $( \pm 1, \pm b)$. Since the next value of $w$ is determined by the current $z$, only certain transitions are permitted, as shown in Fig. 11. The dynamics at the AI limit is conjugate to the full two-shift on sequences of symbols: $\left\{\ldots s_{-1} . s_{0} s_{1} \ldots\right\} \mapsto\left\{\ldots s_{-1} s_{0} . s_{1} \ldots\right\}$, where the binary point denotes the current position.

It is easy to see that this conjugacy extends away from $\epsilon=0$ [7]. In fact there is a one-to-one correspondence between bounded orbits of the Hénon map and symbol sequences $\left\{\ldots s_{t} \ldots\right\}$ when $|\epsilon|(1+|b|) \leq 2 \sqrt{1-2 / \sqrt{5}}$. Moreover, $z_{t} \rightarrow s_{t}$ as $\epsilon \rightarrow 0$ [8].

In previous work we have shown that continuation from the AI limit $(\epsilon=$ 0 ) is an effective tool for studying orbits of the Hénon map [8, 5]. In this section we show how to compute the self-rotation number for orbits at the AI limit and in so doing, define the self-rotation number for a symbolic sequence. Since the Hénon map avoids an angle, this rotation number remains invariant as $\epsilon$ and $b>0$ change. In particular, the self-rotation number remains invariant under continuation away from $\epsilon=0$, the basis for the numerical method presented in [5].

Without loss of generality, we can set $b=1$ since the self-rotation number is independent of positive $b$. Let $v_{t}=f^{t}(z, w)-f^{t-1}(z, w)$, so that the 


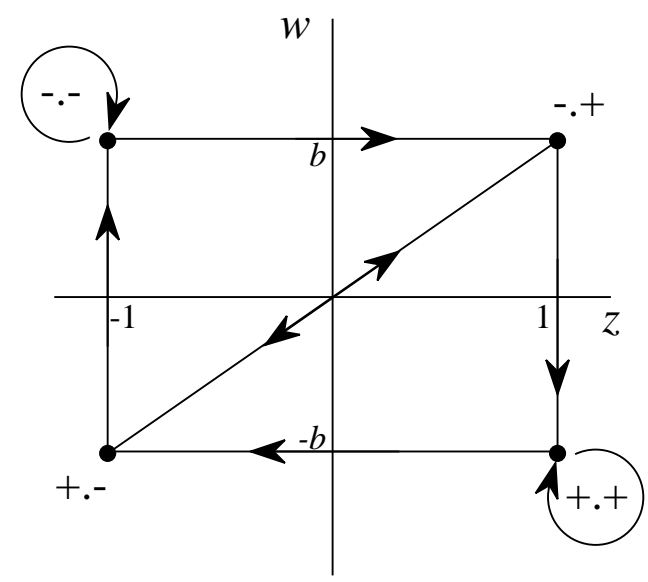

Figure 11: Anti-integrable states for the Hénon map, and the allowed transitions. The corners of the rectangle are labeled by the symbols $s_{t-1} . s_{t}$ of $z_{t}$.

turning angle is $\varphi_{t}=\Phi_{\alpha}\left(v_{t}, v_{t+1}\right)$, with $\alpha=-\frac{3 \pi}{2}$. Whenever the vectors $v_{t}$ are nonzero as $\epsilon \rightarrow 0$, we can easily compute the rotation number by using the turning angle at $\epsilon=0$. This is the case when the symbol sequence does not have more than two contiguous - or + symbols. For this case the turning angle depends only upon four symbols $\left\{s_{t-2} s_{t-1} . s_{t} s_{t+1}\right\}$, since this is sufficient to compute $v_{t}$ and $v_{t+1}$. For example, the symbol sequence $\{\ldots+-.++\ldots\}$ represents the transitions $+.-\mapsto-.+\mapsto+.+$, so that the vector $v_{0}$ corresponds to the diagonal moving from.+- to.-+ , and the vector $v_{1}$ to the vertical moving from.-+ to.++ :

$$
\begin{aligned}
& v_{0}=\left(z_{0}, w_{0}\right)-\left(z_{-1}, w_{-1}\right)=(1,1)-(-1,-1)=(2,2), \\
& v_{1}=\left(z_{1}, w_{1}\right)-\left(z_{0}, w_{0}\right)=(1,-1)-(1,1)=(0,-2),
\end{aligned}
$$

so $\varphi_{0}=-\frac{3 \pi}{4}$. This is precisely the angle seen in Fig. 11. We summarize the turning angles for each transition in Table 1.

The turning angle cannot be directly evaluated at the AI limit when the orbit remains at the points.++ or.-- for more than one iteration. To compute the turning angle in this case, we assume that $0<\epsilon \ll 1$. The sequences $\{\ldots+.+\ldots\}$ and $\{\ldots-., \ldots\}$ correspond to points that are within $\mathcal{O}(\epsilon)$ of the fixed points $z=-w=\epsilon \pm \sqrt{1+\epsilon^{2}}$, respectively. For such points, the dynamics are conjugate to the linearization of the map at the 


\begin{tabular}{c|cccc}
$\left(s_{t-2} s_{t-1}\right) \backslash\left(s_{t} s_{t+1}\right)$ & -- & -+ & +- & ++ \\
\hline-- & $-\pi$ & $\Theta_{n}$ & $-\frac{3 \pi}{4}$ & $-\frac{\pi}{2}$ \\
-+ & $-\frac{3 \pi}{4}$ & $-\pi$ & $-\frac{\pi}{2}$ & $-\frac{\pi}{4}$ \\
+- & $\Theta_{n}$ & $-\frac{\pi}{2}$ & $-\pi$ & $-\frac{3 \pi}{4}$ \\
++ & $-\frac{\pi}{2}$ & $-\frac{3 \pi}{4}$ & $-\frac{\pi}{4}$ & 0
\end{tabular}

Table 1: Turning angles for the Hénon map at the AI limit when $b=1$

fixed points, which is hyperbolic and hyperbolic with reflection, respectively. The multipliers are $\lambda_{u}= \pm \frac{2}{\epsilon}(1 \pm \mathcal{O}(\epsilon))$ and $\lambda_{s}=1 / \lambda_{u}$. The corresponding eigenvectors are $u=( \pm 2-\mathcal{O}(\epsilon), \epsilon)$, and $s=( \pm \mathcal{O}(\epsilon), 2)$, which are basically horizontal and vertical, respectively. Thus the eigenvectors divide the plane into the four quadrants.

A symbol sequence $\left\{\ldots-+^{n}-\ldots\right\}$ stays near the.++ fixed point for $n-1$ iterations. During this time, the orbit lies very close to a branch of the invariant hyperbola of the linearized map. Thus the total turning angle for the $n-1$ iterates from $\left\{\ldots-+.+^{n-1}-\ldots\right\}$ to $\left\{\ldots-+{ }^{n} .-\ldots\right\}$ is $-\frac{\pi}{2}$ as $\epsilon \rightarrow 0$ since the incoming vector $v_{0}=(0,-2)+\mathcal{O}(\epsilon)$, the outgoing vector $v_{n}=(-2,0)+\mathcal{O}(\epsilon)$, and the intermediate vectors monotonically move a quarter clockwise rotation along the hyperbola from $v_{0}$ to $v_{n}$. A consistent choice of turning angles at the AI limit is thus to set $\varphi(++.++)=0$, and to symmetrically allocate half of the total turning to the initial and final points, thus $\varphi(-+.++)=\varphi(++.+-)=-\frac{\pi}{4}$.

By contrast, the -.- fixed point is reflection hyperbolic so that an orbit in an $\epsilon$ neighborhood reflects across the fixed point each iteration, undergoing a turning angle very nearly $-\pi$. A sequence $\ldots+-^{n}+\ldots$, spends $n-1$ iterations near the fixed point with the incoming vector $v_{0}=(0,2)+\mathcal{O}(\epsilon)$, and the outgoing vector $v_{n}=(2,0)+\mathcal{O}(\epsilon)$. If $n$ is even the point $\left(z_{0}, w_{0}\right)$ must be in the $2^{\text {nd }}$ quadrant, so that the final vector will lie along the positive half of the unstable manifold, see Fig. 12. In this case, the vectors $v_{2 i}$ turn monotonically clockwise, so that the ultimate turning angle is $\left(-n+\frac{1}{2}\right) \pi$. If $n$ is odd, however, then the initial point is in the $1^{\text {st }}$ quadrant, and the vectors 
$v_{2 i}$ turn monotonically counter-clockwise, see Fig. 13. Thus the total turning angle in this case is $\left(-n-\frac{1}{2}\right) \pi$. It is consistent to set $\varphi(--.--)=-\pi$ since each iteration at -- corresponds to a half rotation. We symmetrically add an additional rotation of $\pm \frac{\pi}{4}$ to the incoming.+--- , and outgoing .--++ depending upon whether $n$ is even or odd:

$$
\varphi\left(+-.-^{n-1}+\right)=\varphi\left(+-^{n-1} \cdot-+\right)=\Theta_{n} \equiv-\pi+(-1)^{n} \frac{\pi}{4} .
$$

For example, the period 3 orbit, $\{--+\}^{\infty}=\{\ldots--+--+\ldots\}$, corresponds to the sequence of transitions

$$
\begin{aligned}
& --.+-\rightarrow-+.--\rightarrow+-.-+ \\
& -\frac{3 \pi}{4}-\frac{3 \pi}{4} \quad-\frac{\pi}{2}=-2 \pi,
\end{aligned}
$$

so that the rotation number is $-\frac{1}{3}$. A similar computation for the period 4 orbit, $\{---+\}^{\infty}$, gives

$$
\begin{aligned}
& --.-+\rightarrow--.+-\rightarrow-+.--\rightarrow+-.-- \\
& \begin{array}{llll}
-\frac{5 \pi}{4} & -\frac{3 \pi}{4} & -\frac{3 \pi}{4} & -\frac{5 \pi}{4}
\end{array}
\end{aligned}
$$

where we used (17) for the first and last transitions. Here the rotation number is $-\frac{2}{4}=-\frac{1}{2}$ which is consistent with this orbit being born in a period doubling bifurcation of the period two orbit $\{+-\}^{\infty}$ which has the same rotation number.

We list the low period orbits of the Hénon map, their rotation numbers, and bifurcations in Table 2. Knowledge of the rotation number is not sufficient to predict all bifurcations, however, it does give some restrictions. For example only 2 period 5 orbits have rotation number $-\frac{1}{5}$, so they must be born together in a bifurcation. When $b=1$ this bifurcation is a rotational bifurcation of the elliptic fixed point; more generally this will be a saddlenode bifurcation. The situation at period six is more complicated since there are 5 orbits with rotation number $-\frac{2}{6}$. Since this is equivalent to $-\frac{1}{3}$, we suspect that one of the orbits is born by period doubling of a period three orbit. We observe that this is the orbit $\left\{+-{ }^{4}+\right\}^{\infty}$. The next two orbits are asymmetric, and arise from a pitchfork bifurcation of a symmetric orbit when $b=1[5]$. The last two $\rho_{s}=-\frac{2}{6}$ orbits collide in a saddle-node bifurcation. 


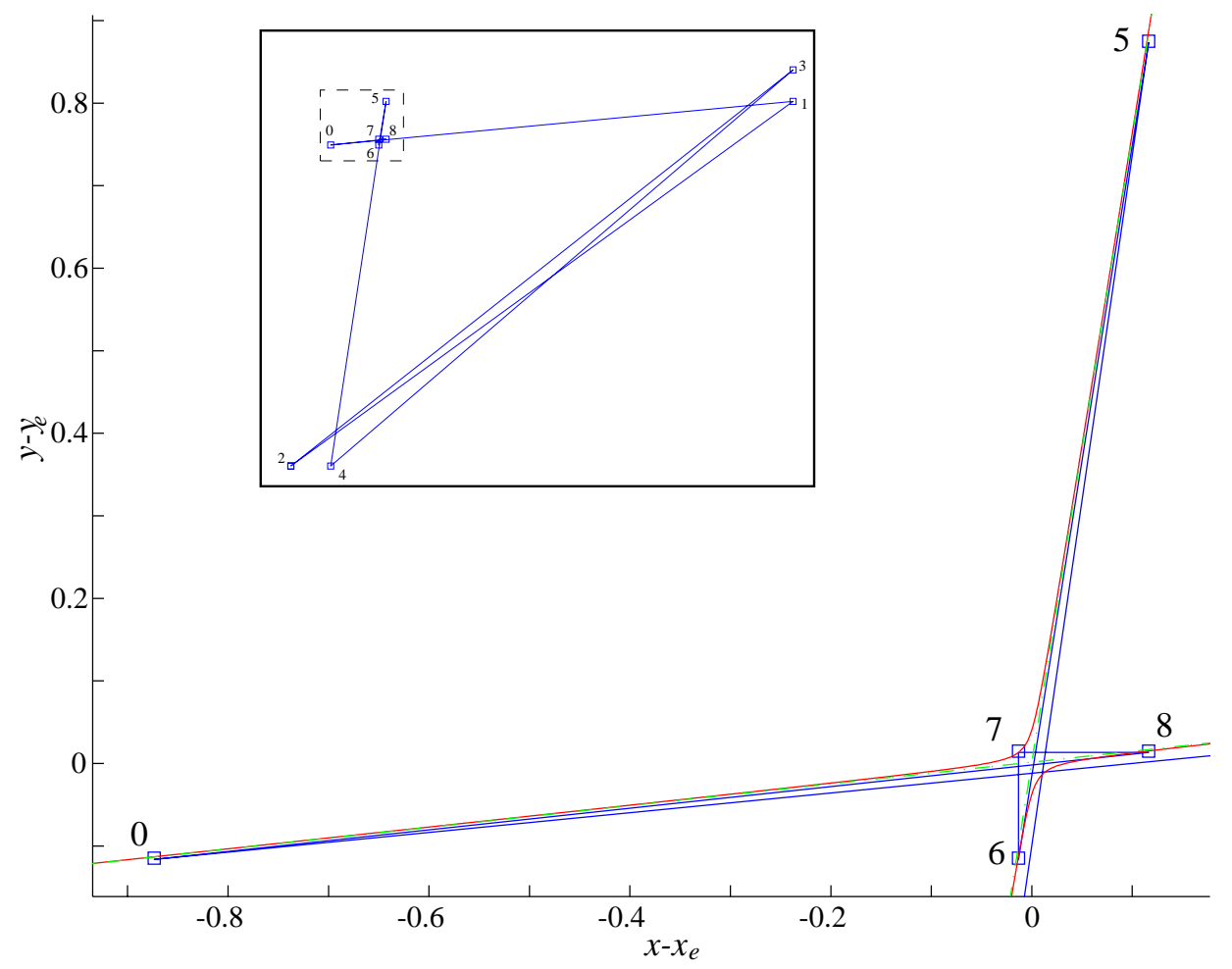

Figure 12: The orbit $\left\{+-{ }^{6}+-\right\}^{\infty}$ for $k=22.7$ and $b=1$. The full period 9 orbit is shown in the inset. The enlargement shows the region centered at the $\{-\}^{\infty}$ fixed point, together with the linear approximations of the manifolds and an invariant hyperbola. The points fall alternately on two branches of the hyperbola because the multipliers of the map are negative, illustrating (17). 


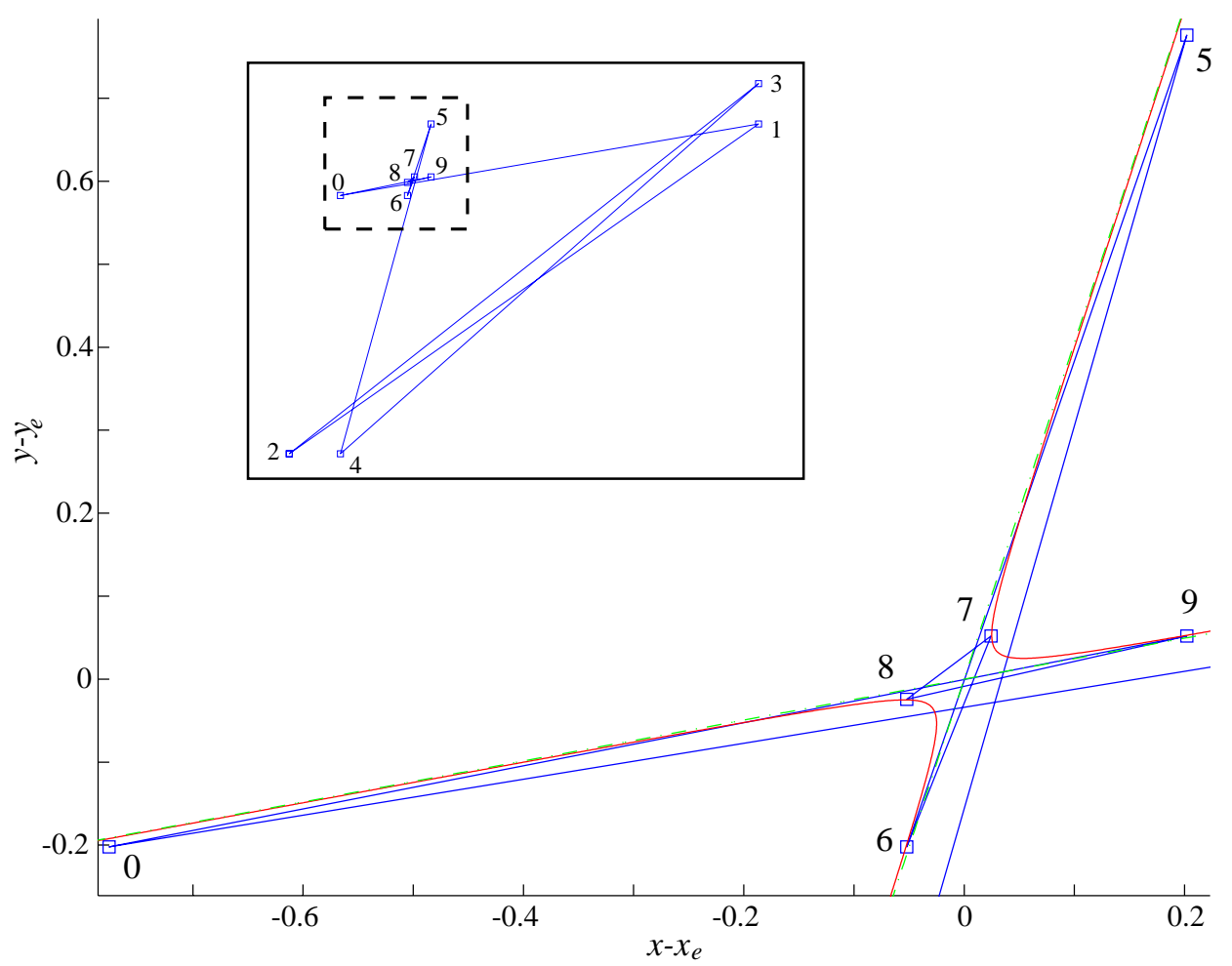

Figure 13: The orbit $\left\{+-{ }^{7}+-\right\}^{\infty}$ for $k=8.92$ and $b=1$. The full period 10 orbit is shown in the inset. The enlargement shows the region centered at the $\{-\}^{\infty}$ fixed point, together with the linear approximations of the manifolds and an invariant hyperbola. The points near the fixed point lie on the hyperbola, but $k$ is not large enough for the approximation to be valid for the two farthest points. 


\begin{tabular}{|c|c|c|c|c|}
\hline Orbit & $\rho_{s}$ & Bifurcation & Parent & $k$-Value \\
\hline$\{+-\}^{\infty}$ & $-\frac{1}{2}$ & $\mathrm{pd}$ & $\{-\}^{\infty}$ & 3 \\
\hline$\{-+-\}^{\infty}$ & $-\frac{1}{3}$ & sn & & 1 \\
\hline$\{+-+\}^{\infty}$ & $-\frac{1}{3}$ & sn & & 1 \\
\hline$\{+--+\}^{\infty}$ & $-\frac{1}{4}$ & $1 / 4$ & $\{-\}^{\infty}$ & 0 \\
\hline$\{+-++\}^{\infty}$ & $-\frac{1}{4}$ & $1 / 4$ & $\{-\}^{\infty}$ & 0 \\
\hline$\{-+--\}^{\infty}$ & $-\frac{2}{4}$ & $\mathrm{pd}$ & $\{+-\}^{\infty}$ & 4 \\
\hline$\{-+++-\}^{\infty}$ & $-\frac{1}{5}$ & $1 / 5$ & $\{-\}^{\infty}$ & -0.52254249 \\
\hline$\{++-++\}^{\infty}$ & $-\frac{1}{5}$ & $1 / 5$ & $\{-\}^{\infty}$ & -0.52254249 \\
\hline$\{--+--\}^{\infty}$ & $-\frac{2}{5}$ & $2 / 5$ & $\{-\}^{\infty}$ & 2.27254249 \\
\hline$\{-+-+-\}^{\infty}$ & $-\frac{2}{5}$ & $2 / 5$ & $\{-\}^{\infty}$ & 2.27254249 \\
\hline$\{+-+-+\}^{\infty}$ & $-\frac{2}{5}$ & $\mathrm{sn}$ & & 5.5517014 \\
\hline$\{+---+\}^{\infty}$ & $-\frac{2}{5}$ & sn & & 5.5517014 \\
\hline$\left\{-+^{4}-\right\}^{\infty}$ & $-\frac{1}{6}$ & $1 / 6$ & $\{-\}^{\infty}$ & $-\frac{3}{4}$ \\
\hline$\left\{++-+^{3}\right\}^{\infty}$ & $-\frac{1}{6}$ & $1 / 6$ & $\{-\}^{\infty}$ & $-\frac{3}{4}$ \\
\hline$\left\{+-{ }^{4}+\right\}^{\infty}$ & $-\frac{2}{6}$ & $\mathrm{pd}$ & $\{+-+\}^{\infty}$ & $\frac{5}{4}$ \\
\hline$\{++-+--\}^{\infty}$ & $-\frac{2}{6}$ & $\mathrm{pf}$ & $\left\{+-{ }^{4}+\right\}^{\infty}$ & 3 \\
\hline$\{--+-++\}^{\infty}$ & $-\frac{2}{6}$ & $\mathrm{pf}$ & $\left\{+-{ }^{4}+\right\}^{\infty}$ & 3 \\
\hline$\left\{+-+^{3}-\right\}^{\infty}$ & $-\frac{2}{6}$ & sn & & 5.6793695 \\
\hline$\left\{--+^{3}-\right\}^{\infty}$ & $-\frac{2}{6}$ & sn & & 5.6793695 \\
\hline$\{--+-+-\}^{\infty}$ & $-\frac{3}{6}$ & sn & & 3.7016569 \\
\hline$\left\{--+-{ }^{3}\right\}^{\infty}$ & $-\frac{3}{6}$ & $\mathrm{sn}$ & & 3.7016569 \\
\hline
\end{tabular}

Table 2: Self-Rotation numbers for periodic orbits of the Hénon map up to period 6 for any $b>0$, and their bifurcations when $b=1$. We do not list the fixed points, since they do not have a fixed self-rotation number. The $3^{\text {rd }}$ column lists the bifurcation that creates the orbit: "sn" indicates a saddle-node, "pf" a pitchfork, and "pd" a period doubling. A rotational bifurcation is denoted by $m / n$, referring to the multiplier of the elliptic point at the bifurcation. For a discussion of these bifurcations see [5]. 
The effectiveness of the rotation number in pruning possible bifurcations decreases with period since the number of orbits grows exponentially, while the number of rotation numbers only grows linearly. We list the number of orbits with each rotation number up to period 16 in Table 3. Some simple observations follow from this table. For example, in general there are only two orbits with self-rotation number $-\frac{1}{n}$, and therefore they must be born together. For the area-preserving case, this occurs at a rotational bifurcation of the elliptic fixed point. These orbits have symbol sequences $\left\{-+^{n-1}\right\}^{\infty}$ and $\left\{--+{ }^{n-2}\right\}^{\infty}$.

Similarly, orbits created in rotational bifurcations from the period two orbit have self-rotation number $-\frac{k}{2 k}$. In particular, the pair of orbits created in the " $1 / \mathrm{k}$ " rotational bifurcation of the period two orbit have sequences $\left\{(--)^{k-2}---+\right\}^{\infty}$ and $\left\{(--)^{k-2}-+-+\right\}^{\infty}$. For period six, since there are only two orbits with rotation number $-\frac{3}{6}$, this means that these orbits must be created together, and in the case $b=1$, that they collide with the period two orbit in a tripling bifurcation. For higher periods, there are more than two orbits with rotation number $-\frac{k}{2 k}$, and so the pruning is not complete.

A final observation is that whenever the number of orbits with given rotation number is odd, at least one of these orbits must be born without a partner, i.e., as a result of a period doubling bifurcation. For example, one of the three orbits with self-rotation number $-\frac{4}{8}$ must be the period doubling of the orbit with rotation number $-\frac{2}{4}$.

\section{Conclusions}

Since the orientation-preserving Hénon map avoids the angle $\alpha=-\frac{3 \pi}{2}$, we can compute the self-rotation number of any orbit using the turning-angle (11) with the arctangent chosen to have the range $(\alpha, \alpha+2 \pi)$. The selfrotation number can be computed at the anti-integrable limit using the symbol sequence for an orbit. This encoding is equivalent to that for the standard horseshoe. Since the self-rotation number is preserved upon variation of the parameters $k$ and $b$, orbits with different self-rotation numbers can never coincide, and so they cannot be partners in a bifurcation. This conclusion 


\begin{tabular}{|c|c|c|c|c|c|c|c|c|c|}
\hline \multicolumn{2}{|c|}{ Orbits } & \multicolumn{7}{c|}{ Self-rotation Number $\left(\rho_{s}\right)$} \\
\hline period (n) & total \# & $-1 / \mathrm{n}$ & $-2 / \mathrm{n}$ & $-3 / \mathrm{n}$ & $-4 / \mathrm{n}$ & $-5 / \mathrm{n}$ & $-6 / \mathrm{n}$ & $-7 / \mathrm{n}$ & $-8 / \mathrm{n}$ \\
\hline 2 & 1 & 1 & & & & & & & \\
3 & 2 & 2 & & & & & & & \\
5 & 3 & 2 & 1 & & & & & & \\
6 & 6 & 2 & 4 & & & & & & \\
7 & 9 & 2 & 5 & 2 & & & & & \\
8 & 18 & 2 & 8 & 8 & & & & & \\
9 & 30 & 2 & 9 & 16 & 3 & & & & \\
10 & 96 & 2 & 12 & 26 & 16 & & & & \\
11 & 186 & 2 & 16 & 56 & 80 & 32 & & & \\
12 & 335 & 2 & 17 & 74 & 137 & 96 & 9 & & \\
13 & 630 & 2 & 20 & 96 & 224 & 224 & 64 & & \\
14 & 1161 & 2 & 21 & 120 & 332 & 448 & 220 & 18 & \\
15 & 2182 & 2 & 24 & 146 & 480 & 806 & 596 & 128 & \\
16 & 4080 & 2 & 25 & 176 & 655 & 1344 & 1336 & 512 & 30 \\
\hline
\end{tabular}

Table 3: Winding Numbers for periodic orbits of the Hénon map with $b>0$. The total number of periodic orbits is the number for a full 2-shift. 
requires $b>0$, thus the restrictions on the allowed bifurcations do not continue to the one-dimensional limit.

We have shown that any orientation preserving linear map avoids an angle $\alpha$, and so nearly linear maps will avoid that angle as well. It is not clear, however, how general this concept is. For example, numerical evidence indicates that the standard map (see e.g., [2]) avoids an angle, however, the proof of this appears non-trivial.

To compute the rotation numbers of orbits born in rotational bifurcations about elliptic periodic orbits (i.e., islands-around-islands), it would be useful to know when $f^{n}$ avoids an angle as well. For example, suppose that the rotation number of a period $q_{0}$ orbit is $\frac{p_{0}}{q_{0}}=\frac{q_{1} m_{0}}{q_{1} n_{0}}$, where $m_{0}$ and $n_{0}$ are coprime. Then the period $q_{0}$ orbit may be in the bifurcation tree generated by a period $n_{0}$ orbit with rotation number $\frac{m_{0}}{n_{0}}$. To determine the rotation number relative to the period $n_{0}$ orbit, we could compute the self-rotation number using the map $f^{n_{0}}$, giving $\frac{p_{1}}{q_{1}}$ for some $p_{1}$. This process can be continued until $p_{k}$ and $q_{k}$ are coprime. Ultimately the rotation number would be expanded in a complete sequence $\frac{m_{0}}{n_{0}}: \frac{m_{1}}{n_{1}}: \ldots: \frac{m_{k}}{n_{k}}$, so that $\prod n_{i}=q_{0}$.

Finally, though we have only discussed planar maps in this paper, it would be nice to generalize the concept of self-rotation number to higherdimensional maps. For example, for the class of symplectic maps, the symplectic structure gives special meaning to canonical pairs that may make the concept of rotation vector meaningful. 


\section{References}

[1] A. Katok and B. Hasselblatt. Introduction to the Modern Theory of Dynamical Systems. Cambridge University Press, Cambridge, 1995.

[2] J.D. Meiss. Symplectic maps, variational principles, and transport. Reviews of Modern Physics, 64(3):795-848, 1992.

[3] K.R. Meyer and G.R. Hall. Introduction to the Theory of Hamiltonian Systems, volume 90 of Applied Mathematical Sciences. Springer-Verlag, New York, 1992.

[4] B. B. Peckham. The necessity of the hopf bifurcation for periodically forced oscillators. Nonlinearity, 3(2):261-280, 1990.

[5] D. Sterling, H.R. Dullin, and J.D. Meiss. Homoclinic bifurcations for the Hénon map. Physica D, 134:153-184, 1999.

[6] H.R. Dullin and J.D. Meiss. Generalized Hénon maps: the cubic diffeomorphisms of the plane. Physica D, 2000.

[7] S. J. Aubry. Anti-integrability in dynamical and variational problems. Physica D, 86:284-296, 1995.

[8] D. Sterling and J.D. Meiss. Computing periodic orbits using the antiintegrable limit. Physics Letters A, 241:46-52, 1998. 